\title{
Reconquête culturo-économique des territoires délaissés : de l'importance du temps de veille et de ses acteurs transitoires
}

\author{
Lauren Andres \\ Dr en urbanisme et aménagement \\ Lecturer in Spatial Planning \\ Centre for Urban and Regional Studies, Birmingham Business School \\ University of Birmingham, Edgbaston, Birmingham, B15 2TT, UK \\ L.Andres@Bham.ac.uk
}

Chercheuse associée à l'UMR Telemme (Aix en Provence)

Article publié dans Méditerranée, ${ }^{\circ} 114$, pp. 51-64

\section{Introduction}

Les espaces délaissés dans les villes d'aujourd'hui, et, plus particulièrement, les espaces caractérisés comme friches urbaines suscitent nombre d'interrogations et de représentations car ils constituent des espaces à part dans la ville. Une des particularités des friches urbaines réside dans le fait que, outre leurs états d'abandon et de non-usage apparent, elles renvoient à une utilisation passée et laissent présager, à court, moyen et long terme, une transformation plus ou mois brutale et respectueuse de l'existant; de manière sous-jacente, trois temps se succèdent et se confrontent au cours de la trajectoire de mutation dans laquelle ces espaces vont s'insérer : le temps de l'avant-friche (héritage et squelette en quelque sorte), celui de la friche proprement dit et enfin le temps de l'aprèsfriche (phase de projections et de projet). Interroger la reconquête culturo-économique de ces espaces délaissés implique d'insister sur le temps de friche, c'est-à-dire sur le temps de veille, dont les caractéristiques sont développées ci-après. Ce temps de veille est propice au développement de nouveaux usages impliquant de fait des rapports paradoxaux et variables, entre les propriétaires de friches, les acteurs publics et les acteurs culturels ou économiques s'installant sur ces espaces de manière temporaire dans l'attente d'une réutilisation future, d'où leur qualificatif d'acteurs transitoires. L'intérêt d'une étude approfondie du temps de veille de la friche, des pratiques qui s'y développent et des stratégies qui y sont sous-jacentes, est une manière à mon sens d'analyser et de décrypter la mutation de ces territoires à part, qui ne sont en aucun cas des squats ; c'est aussi une façon d'insister sur la capacité de cette période à être le levier de transformations futures conduisant in fine à la réinscription des friches dans l'espace urbain. La friche est ainsi comparable à un « espace intermédiaire » (ROULLEAU BERGER, 1991 ; 1995 ; 1999), un 
« interstice urbain » (HATZFELD, HATZFELD, RINGART, 1998) / un « espace indéterminé » (GROTH, CORIJN, 2005), un « espace temporaire urbain » (HAYDN, TEMEL, 2006) ou encore une « zone libre » (URBAN UNLIMITED, 2004). L'acteur transitoire tend à y être producteur de ressources mais aussi créateur de processus de renouvellement.

Cet article vise donc à s'interroger sur l'importance que peut prendre le temps de veille dans la requalification des espaces délaissés et, en particulier, sur l'influence qu'il peut avoir sur la construction d'un projet de renouvellement urbain. Par ailleurs, il aspire aussi à insister sur les systèmes d'acteurs en présence et surtout sur les conditions permettant à des acteurs, au statut initialement transitoire, de pénétrer les sphères relativement fermées de la mise sur agenda et de la construction des projets urbains. Deux cas de figure, atypiques et non-reproductibles certes mais particulièrement riches vis-à-vis des résultats qu'ils suscitent, alimentent cette réflexion (ANDRES, 2008): la Friche de la Belle de Mai à Marseille et la plateforme du Flon à Lausanne. La Friche de la Belle de Mai est une ancienne manufacture des tabacs réutilisée dès 1991 par des acteurs culturels (association Système Friche Théâtre), insérée dans le projet Euroméditerranée en 1995 et faisant désormais partie d'un pôle culturel composé de trois îlots (1-patrimoine /2- industries culturelles / 3spectacle vivant, en d'autres termes La Friche proprement dite devenue une SCIC en 2007). La plateforme du Flon à Lausanne, quant à elle, est une ancienne plateforme de stockage créée à la fin du $19^{\mathrm{ème}}$ siècle et sous-utilisée depuis les années 1950. L'impossibilité de parvenir à la votation d'un nouveau plan d'affectation (avant 1999), condition sine qua non pour la modification de la fonction industrielle du site, et ce du fait de nombreux conflits et jeux d'intérêt entre le propriétaire du site (le Lo Holding), la Ville et la population locale, amène la transformation par le bas du Flon suite à l'arrivée de petits acteurs économiques et culturels attirés par des bâtiments, au cœur de la ville, modulables et peu chers à la location. D'une situation marginalisée, le site (nommé alors "Flon-Flon ») retrouve toute son importance comme pôle de centralité ; la convergence des nouvelles stratégies de développement du Lo (Flon Vision) et des politiques de développement du centre-ville (via un Plan partiel d'Affection voté en 1999) de la municipalité mène à la constitution d'un quartier certes toujours atypique mais régénéré et par delà gentrifié économiquement car jointif à la nouvelle plateforme multimodale des transports publics de la ville.

Dans un premier temps, seront ainsi examinées les spécificités des friches urbaines durant leur temps de veille et des activités qui s'y développent puis les conditions de la transition entre temps de veille et temps de l'après-friche. Enfin, un regard critique sera présenté sur la normalisation progressive de ces espaces urbains et leurs impacts sur les tissus environnants. 


\section{1 - La friche en veille : un espace permissif propice aux expérimentations}

La friche en veille est en espace accessible dont le tissu et la morphologie sont propices à des nouvelles utilisations, plus ou moins pérennes. En d'autres termes, ce ne sont pas des espaces en ruine ou de vieux hangars délabrés mais le plus souvent d'anciens bâtiments industriels propices aux transformations et à une souplesse quant aux nouveaux usages qui peuvent s'y développer. Typiquement, l'ancienne manufacture des tabacs à Marseille comprend différent types de bâtis : des entrepôts, des bâtiments en dur de plusieurs étages (comme illustré sur la photo 1), des maisonnettes, aptes à fournir ateliers, studios de répétition, salles de spectacles, etc., puisqu'ils constituent, selon F. Lextraitii, " un potentiel de jeu et d'exploration incroyable ". De même le Flon est constitué d'anciens entrepôts en dur de deux ou trois étages offrant également des espaces de stockage importants en soussols. Ses formes géométriques sont propices à des réaménagements extrêmement variés, l'espace intérieur étant modulable (cf. photo 2).

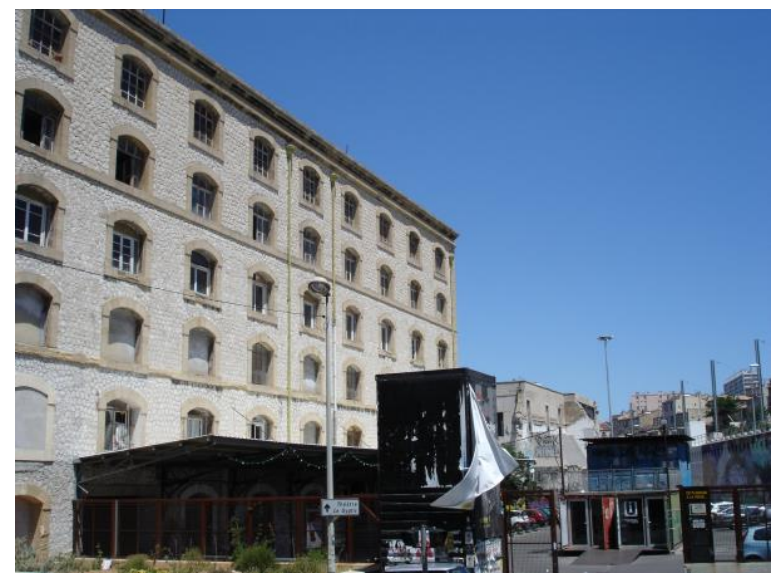

Photo 1 : La Friche de la Belle de Mai et son bâtiment-lieu de travail principal (L. Andres, 2008)

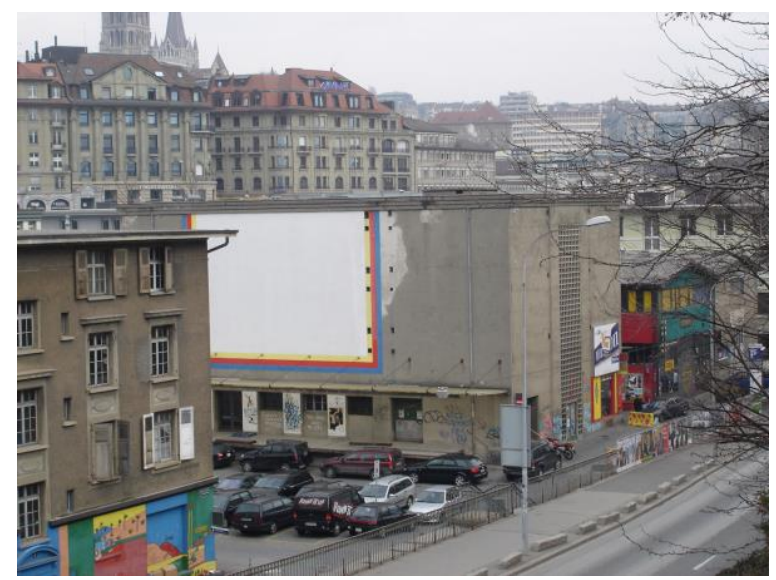

Photo 2 : Les hangars du Flon (L. Andres, 2007) 
Par ailleurs, la friche en veille est aussi un espace à part dans la ville. Par sa situation enclavée, en bordure, "à la fois poubelle ou exutoire, et laboratoire, promesse d'états meilleurs » (HATZFELD, HATZFELD, RINGART, 1998, p.15), elle est favorable à l'émergence de nouvelles pratiques et réappropriations. La manufacture de la Belle de Mai jouxte la voie ferrée et se situe en bordure et en position surélevée par rapport au reste du quartier (voir photo 3); de même la plateforme de Flon est en contrebas $(12 \mathrm{~m})$ du centre-ville de Lausanne, donc à l'écart (cf. photos 2 et 4). Les deux caractéristiques majeures de ces espaces interstitiels résident, d'une part, dans leur permissivité, allant de pair avec un retrait des acteurs publics, et, d'autre part, dans leur " turbulence » (HATZFELD, HATZFELD, RINGART, 1998) : ils abritent diverses activités et une multitude d'acteurs dont les enjeux et les relations changent rapidement. Leur ambiance et leur physionomie sont ainsi une clé pour caractériser ses espaces comme c'est le cas par exemple au Flon au début des années 1990 comme l'illustre le propos d'un des locatairesiii: " C'était un quartier un peu atypique, pas trop en ordre, pas suisse quelque part, (...) il régnait une anarchie (...) contrôlée (...) ».

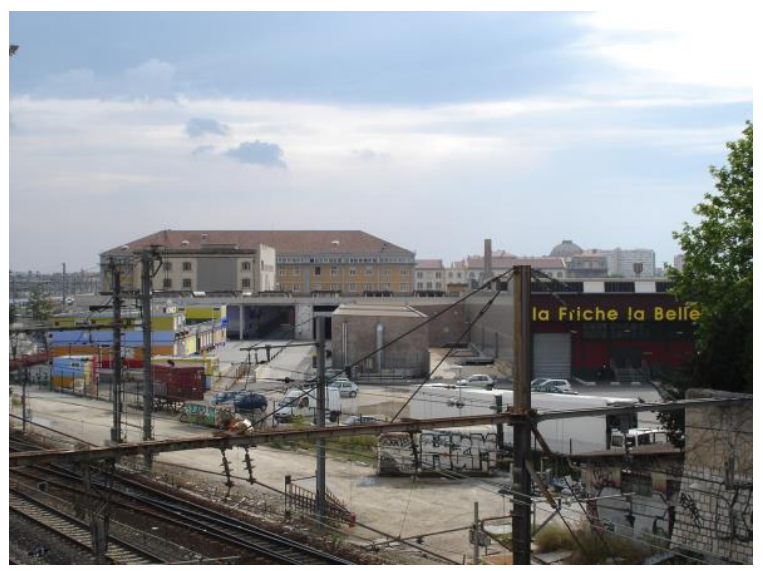

Photo 3 : La Friche et l'îlot 2 en bordure de la voie ferrée et en haut d'une butte (L. Andres, 2008)

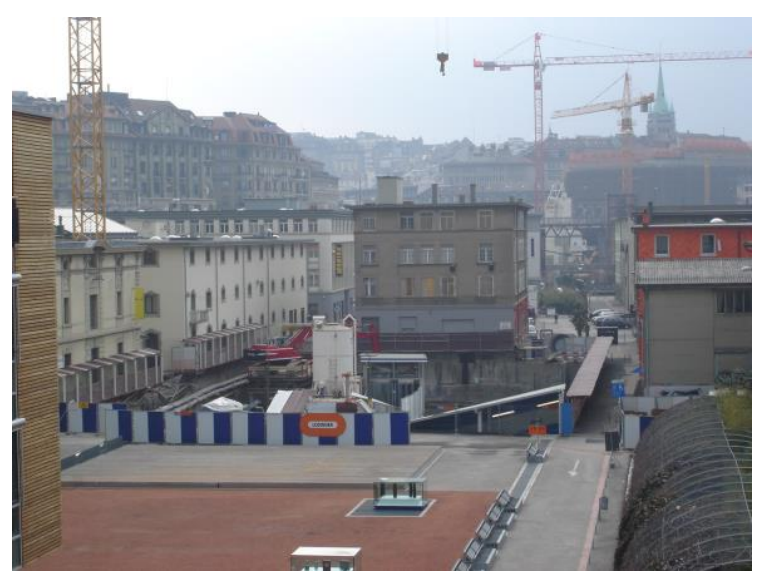

Photo 4 : Le Flon 123 au-dessous du centre-ville (L. Andres, 2008)

Cette permissivité est synonyme de non-conformisme; comme le rappelle P. OSWALT (2005), ceci n'est pas anodin sachant que ces espaces urbains délaissés ont vu naître nombreux mouvements de contre-culture ou de culture alternative : la techno à Détroit ou 
la musique punk, la techno et l'house music, à Manchester et Sheffield, à la fin des années 1970. Par là même, comme souligné par H. HATZFELD, M. HATZFELD, N. RINGART (1998), leur caractère de turbulence les rend propices à différents formes d'appropriations, à la fois culturelles et économiques. Typiquement, ces nouvelles fonctions et activités vont s'inscrire dans ce que L. ROULLEAU BERGER $(1995,2002)$ nomme les mondes de la petite production sociale et économique. A Lausanne, au delà de l'intérêt porté au site fondé sur ses loyers avantageux, sur sa localisation et la modularité des bâtiments, les nouveaux locataires sont attirés par le caractère à part du Flon d'autant que le propriétaire leur laisse une liberté presque totale quant à l'utilisation du site et à l'aménagement des locaux. C'est en ce sens que des commerçants (antiquaires, restaurateurs, responsables de magasins de vêtement, de chaussure ou encore de musique, ...), des architectes, des galeristes spécialisés dans l'art contemporain, des artistes, des musiciens, ou encore des tenanciers de boîtes de nuit, s'installent au début des années 1990 sur la plate-forme. A Marseille, la Friche compte de multiples structures et groupes comme l'hebdomadaire Taktik, la Radio Grenouille, le restaurant La Mezzanine, les danseurs du groupe Dunes ou l'association Aide aux Musiques Innovatrices (AMI). II est certain que les activités qui se développent dans ces espaces intermédiaires sont de diverses natures et se recoupent par la fragilité de leur existence et les faibles moyens financiers dont elles disposent (tout au moins au début). Comme l'a montré L. ROULLEAU BERGER (1995), ces activités peuvent dans certains cas articuler valeurs et pratiques marchandes et non marchandes, et se concentrent sur l'échelle microlocale et locale. Néanmoins, au Flon comme à la Belle de Mai, ces activités ne s'exercent pas qu'à la seule échelle locale, ce qui est une des clés et conditions de la mutation développée ci-après. De fait, les nouvelles formes d'occupation et d'utilisation des espaces modifient et transforment ces territoires. Se développent de nouvelles formes de temporalités, ce qui conduit à une reterritorialisation de la friche et à la création d'une nouvelle urbanité ; cellesci vont clairement influencer la mutation de ces espaces tout comme leurs relations avec les quartiers environnants.

De fait la friche en veille n'est pas un espace hors-temps. Au contraire, les activités nouvellement implantées redonnent une vie et de fait une temporalité à ces espaces ; cette temporalité diffère certes de la temporalité initiale du site puisque après des rythmes réguliers fondés sur les horaires de travail des ouvriers et des manutentionnaires, l'espace est désormais marqué par une utilisation à la fois diurne et nocturne (concerts, boîtes de nuits, ...). Cela conduit à l'afflux de nouvelles personnes dans ces lieux, auparavant nonpratiquantes, et en même temps amène, comme à la Belle de Mai, certains conflits avec la population locale qui n'apprécie guère ces nouvelles nuisances sonores. Parallèlement, les nouvelles fonctions de la friche, ses nouveaux usages mais aussi la nouvelle utilité qui lui est 
donnée conduisent à sa reterritorialisation dans l'espace urbain. L'espace délaissé désormais occupé - réacquiert un rôle, certes non définitif et pérenne mais néanmoins identifiable : le Flon, jusqu'à présent quartier mis à l'écart et oublié renaît grâce aux nouvelles activités qui s'y développent ; il redevient attractif pour la population lausannoise qui n'hésite plus à y descendre; de la même manière l'ancienne manufacture, auparavant espace réservée aux seules personnes habilitées à venir y travailler devient, petit à petit, un espace un peu plus ouvert et traversant pour celles et ceux qui osent franchir le seuil de l'ancienne usine. Ces espaces acquièrent une nouvelle urbanité qui n'est plus celle d'un espace vide, celle d'un territoire industriel et monofonctionnel déconnecté des tissus environnants. Pour autant, par son caractère permissif, l'espace ne s'en trouve pour le moins pas normalisé ; il garde son côté alternatif et reste un espace mineur vis-à-vis d'espaces majeurs environnants, comme l'évoque H. HATZFELD, M. HATZFELD, N. RINGART (1998), il reste "en dehors de la continuité régulière ". D'où des qualificatifs de laboratoire et de lieu d'expérimentation (ROULLEAU BERGER, 1991. 1995 ; 1999 ; HAYDN, TEMEL, 2006 ; OVERMEYER, 2007) ; sont ainsi questionnées les formes traditionnelles d'aménagement et de planification (GROTH, CORIJN, 2005) et réinterrogées les manières de fabriquer et de renouveler la ville, d'où d'ailleurs les différentes études et rapports, soulignant l'intérêt croissant des acteurs publics et de l'Union européenne en particulier (URBAN UNLIMITED, 2004; HAYDN, TEMEL, 2006 ; OVERMEYER, 2007).

Il convient en ce sens de considérer que la friche en veille acquiert une importance stratégique dans l'aménagement urbain qui en fait un espace en aucun cas oublié et déconnecté de l'action publique. Même si les activités qui s'y développent relèvent d'un effet d'opportunité, à la fois économique et politique, les appropriations menées sur une friche de veille ne sont pas spontanées. Elles font clairement l'objet d'un accord préalable entre les propriétaires, les acteurs publics (dans certains cas) et les nouveaux utilisateurs / locataires (acteurs transitoires). Typiquement l'occupation de la manufacture de la Seita est le résultat d'un rapport gagnant-gagnant entre tous les acteurs en présence. Elle s'inscrit dans un contexte de crise. Marseille dans les années 1980-90 est une ville en crise qui souffre d'un marché immobilier en berne (tertiaire en particulier) et d'un déclin économique et démographique (SAN MARCO, MOREL, 1988 ; DONZEL, 1998 ; DUBOIS, OLIVE, 2004)). La Seita propriétaire des lieux, faute de pouvoir vendre son site ou d'y développer une zone d'activité, n'est ainsi pas en mesure de dégager un quelconque profit de ce tènement. Accepter et signer une convention d'occupation précaire avec les acteurs culturels est donc une manière d'entretenir et de sécuriser le site à moindre coût dans l'attente d'une revente future. La Ville, quant à elle, et son adjoint aux politiques culturelles, Christian Poitevin (alias le poète Julien Blaine), prennent le parti de promouvoir une 
approche d'utilisation temporaire des friches (principe de nomadisme) visant à requalifier, grâce à la culture, ces territoires, ou tout au moins à lutter contre les impacts de ces lieux vides sur les quartiers environnants. Les acteurs culturels quant à eux saisissent l'opportunité offerte d'occuper les friches et tout particulièrement la liberté d'action ainsi offerte sur plusieurs hectares, véritables terrains de jeu et d'expérimentation. Le positionnement initial de C. Poitevin est précurseur à l'attention portée au développement de pratiques temporaires sur ces espaces en Europe, et, par exemple à Berlin : "For some time and with increasing frequency, temporary use has turned up in the discourse on planning as a new concept, as a new urban planning method. This temporary uses fall outside of the normal economy. In order to create room for such activities anyway, more and more frequently, particularly in the context of art and culture, legally precarious situations are being tolerated that on the basis of their contracts alone represent only temporary use " (HAYDN, TEMEL, 2006, p. 25). C'est donc véritablement vis à vis d'une situation de non-choix dans la sélection de scenarios plus traditionnels que les artistes et acteurs culturels prennent place sur cet espace. Il en est de même au Flon puisque les locataires du Flon-Flon peuvent investir les anciens entrepôts une fois que le propriétaire a décidé de cesser d'attendre qu'un nouveau plan d'affectation soit voté (des réflexions étant menées depuis les années 1970 - aboutissant d'ailleurs à l'échec, par référendum, d'un premier projet en 1986) ; I'obtention d'une rente financière minimale devenant essentielle, il choisit de louer ses locaux à de petits acteurs économiques et culturels.

II n'en demeure par moins qu'une des questions clés, dans le cas du temps de veille, est la manière dont est appréhendé le passage du temporaire au durable ou, tout au moins, d'une requalification alternative à un vrai projet de mutation. Au Flon comme à la Friche, les activités développées durant le temps de veille modifient considérablement l'image du site et sont des pré-requis à sa requalification; clairement, leur amorce de mutation a un effet levier sur la façon dont un processus de planification plus encadré va alors être conduit. A Marseille, la Friche de la Belle de Mai acquiert très vite le statut d'équipement vitrine pour la ville tant le lieu se construit très rapidement une renommée locale, nationale et européenne. II est d'ailleurs délibérément inséré dans le projet Euroméditerranée en 1995 comme partie prenante des équipements de valorisation «indispensables pour créer l'image de l'opération et provoquer l'effet d'entraînement nécessaire à son démarrage " (MINISTERE DES AFFAIRES SOCIALES, MINISTERE DE L'INTERIEUR ET DE L'AMENAGEMENT DU TERRITOIRE, MINISTERE DE L'EQUIPEMENT, 1993). Au Flon, I'implantation de commerces alternatifs et de boîtes de nuit donne un caractère branché au lieu, de nuit comme de jour. Le Flon est comparé à un petit Soho (PECLET, 1994) et la permissivité du lieu devient une image de marque. Clairement donc, le positionnement et les actions menées 
par les acteurs transitoires sont au cœur du renouvellement de ces sites et il est nécessaire d'en dépeindre les caractéristiques.

Pour désigner ces acteurs qui s'approprient idéellement, symboliquement et physiquement ces lieux, différents termes sont utilisés : J. GROTH et E. CORJIN (2005) parlent d' «acteurs informels », P. OSWALT (2005) et K. OVERMEYER (2007) de " pionniers de l'espace " ou encore F. HAYDN et R. TEMEL (2006) d'«usagers temporaires». Le terme d'acteurs transitoires, en français, parait pertinent pour caractériser ces acteurs non institutionnels, issus de la société civile et exclus des sphères traditionnelles de l'aménagement urbain (politique et techniciste). Ces acteurs construisent un imaginaire et élaborent des stratégies de reconquête sur ces espaces. A la Belle de Mai, ce sont des acteurs culturels et des artistes. Au Flon, ce sont des commerçants, des artisans, des architectes, ...Le qualificatif de « transitoires " permet d'insister sur le caractère temporaire de leur situation et sur le fait que leur place est destinée à évoluer tout comme celle de la friche : certains vont disparaître, d'autres vont voir leur statut se modifier via l'obtention d'un rôle cadré, normé voir institutionnel dans le processus de mutation). Le terme transitoire permet donc de souligner la non-pérennité des formes d'appropriation et de réutilisation qui en découlent et la ré-interrogation progressive de la place et du rôle de ces acteurs transitoires dans le temps de l'après-friche. La pérennité des activités développées durant le temps de veille dépend en effet de facteurs exogènes sur lesquels les acteurs transitoires n'ont qu'une faible prise d'autant plus s'ils n'y associent pas une véritable de stratégie d'assise territoriale et de visibilité, comme développé ci-après: entrent en effet en ligne de compte les stratégies foncières et financières du propriétaire des lieux et celles des acteurs publics en matière d'aménagement et de politiques urbaines. Or, il est clair que les ententes entre les acteurs transitoires et les acteurs décisionnaires et-ou propriétaires, évoquées précédemment, durant le temps de veille, ne sont que provisoires. Le passage dans le stade de l'après-friche conduit à une délicate articulation entre les différents acteurs en présence (institutionnels et non institutionnels) et entre les pratiques spatiales qui leurs sont associées.

\section{Quand les fondements du temps de veille deviennent corps d'une mutation planifiée et normée}

Insister sur l'importance du temps de veille implique de souligner que les activités s'installant sur ces espaces en friche ne demeurent pas temporaires et précaires. Au contraire, elles s'inscrivent comme le corps d'une mutation planifiée et progressivement normalisée (menant de fait à la disparition de l'apparence de friche n'allant néanmoins pas 
de pair avec la disparition de ses utilisateurs en tant que tels). A Marseille, la transition entre le temps de veille et celui de l'après-friche est segmenté et progressif. En effet, outre l'investissement de l'ancienne manufacture en 1991 par les acteurs culturels, il convient de noter aussi que dès 1992 la Ville de Marseille et la DRAC PACA s'intéressent à une autre partie de la manufacture, la plus historique, dans laquelle ils décident d'implanter les archives municipales et un CICRPiv ( ces deux établissement ouvriront respectivement en 2001 et 2002). A ce projet s'ajoute aussi dans la partie centrale de la manufacture, comme illustré sur la figure 1, suite à son insertion dans le projet Euroméditerranée, la création d'un pôle dédié aux activités culturelles et piloté par l'établissement public (EPAEM) en charge de cette opération d'intérêt national (OIN). Seule la Friche reste un peu à l'écart de ces processus de mutation d'envergure allant de pair avec de larges investissements financiers ; son existence n'est jamais remise en cause pour autant mais il faut attendre 1998 pour que le site devienne finalement propriété publique et 2002 pour qu'un véritable projet de mutation soit réalisé.

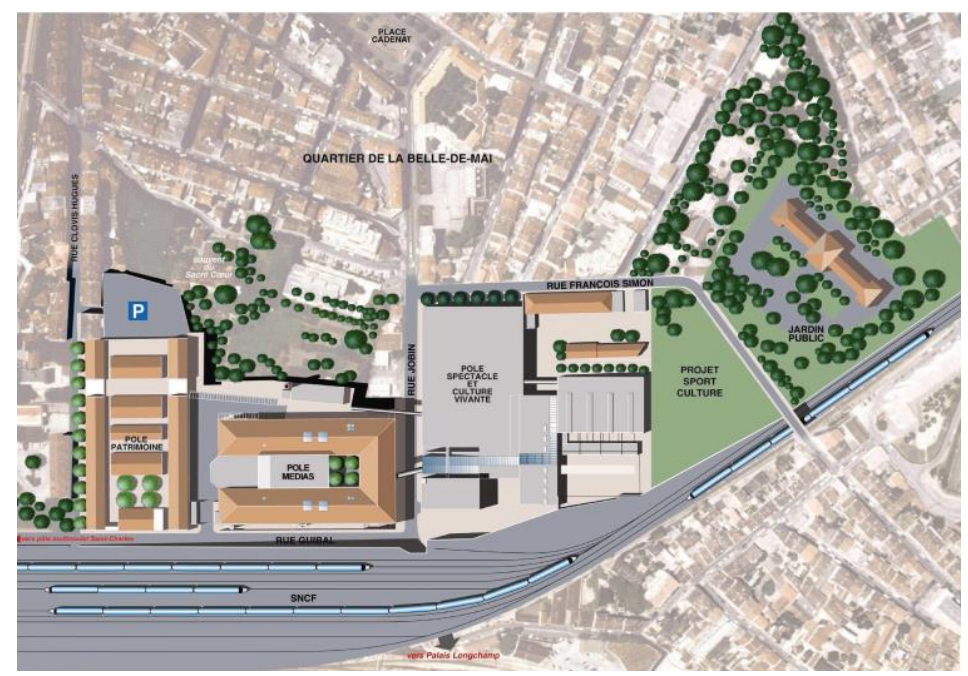

Figure 1 : Les trois pôles culturels de la Belle de Mai (Euroméditerranée, 2007)

Au Flon, le passage du temps de veille à celui de l'après-friche est synonyme de la votation d'un plan partiel d'affectation en 1999 qui met un terme à l'impossibilité de faire muter fonctionnellement et physiquement l'ancienne plateforme de stockage. Le plan est accepté sans aucune opposition à partir du moment où il appelle une mutation du Flon fondée sur ses atouts intrinsèques : sa localisation et ses caractéristiques, tant morphologiques, qu'urbaines et fonctionnelles. Ce plan préserve "la double vocation de quartier à la fois parfaitement central et d'endroit un peu à part, avec son cachet particulier, son style et sa propre façon d'évoluer » (GROUPE LO, 1998). Néanmoins, il offre une très grande flexibilité et liberté d'action au propriétaire au travers de la stratégie de développement (Flon Vision) qu'il met en œuvre. Cela conduit à une modification du Flon qui n'est plus du tout un espace 
à part tant par sa morphologie que par son ambiance. Cette transformation n'est pas forcément au goût des acteurs transitoires : certains quittent le Flon, d'autres tentent de se rebeller, sans aucun résultat.

Dès lors, cette forme de pérennisation de l'affectation fonctionnelle du site (pour partie au moins) et de ses acteurs (en grande majorité) passe par leur prise de conscience (quasi immédiate) de la nécessité de construire une véritable stratégie de développement, tirant profit de l'effet levier et de l'image du site acquis durant le temps de veille. En effet, à Marseille comme à Lausanne, le temps de veille a été propice à la construction d'une véritable friche-vitrine. En d'autres termes, le renouvellement - à part et singulier - est considéré comme un succès et devient un véritable emblème de processus de régénération alternatif pour les acteurs publics et privés. Typiquement, à Marseille, cela se traduit dans le soutien politique et financier constant de la Ville, dont le lieu fait l'objet, tout comme d'ailleurs dans son insertion dans le projet Euroméditerranée. Au Flon, la nouvelle image et la nouvelle attractivité du site, véhiculées par les médias et la population, rend les acteurs du Flon-Flon partie prenante de la mutation, au cœur des négociations avec la Ville, et surtout le propriétaire, au moment de la préparation du PPA de 1999. Cela explique d'ailleurs aussi que les fondements du schéma directeur (1997) et du PPA reposent sur les caractéristiques du Flon-Flon. Cette stratégie de développement passe par un changement d'échelle puisqu'il ne s'agit plus d'une réflexion micro-locale à l'échelle du bâtiment et de la friche mais à l'échelle de la ville.

Le changement d'échelle dans le positionnement de ces acteurs est signe de la prise de conscience de la nécessité de mettre en œuvre une stratégie pour perdurer sur cet espace et y bâtir un réel projet. Cette stratégie peut être anticipée sur du long terme ou s'exprimer de manière revendicative à plus court terme. L'exemple de la Belle de Mai est typique de la démarche d'anticipation stratégique mise en œuvre par SFT dès son installation à La Friche. Elle passe par la constitution d'un socle stable dès l'investissement des lieux : en d'autres termes, outre l'aval de la ville, une entente avec la SEITA et la signature d'une convention d'occupation précaire ; comme l'évoque F. Lextrait ${ }^{v}$, cela était une condition sine qua non: "On a toujours été en règle. Ça nous a été très favorable d'avoir une convention signée avec la Seita parce que ça nous a permis d'avoir les financements de la Ville, ça a mis le projet dans le registre institutionnel dans le sens où on n'était pas des marginaux ou des squatteurs, des alternatifs. Donc, il y a toujours eu une préoccupation très attentive sur le respect de l'essentiel des règles. Ça a qualifié le projet et l'a légitimé. On n'était pas dans un endroit où l'on faisait n'importe quoi. On a toujours lutté contre l'image de squat, l'image underground ". A cela s'ajoutent (1) la création d'une structure porteuse : I'association SFT avec des référents clés ( $P$. Foulquié en tant que directeur, $F$. Lextrait en tant 
qu'administrateur général, auquel s'ajoutent un comptable, un chargé de communication, etc); (2) la constitution d'un projet de développement autour de la culture alternative économique : "l'artiste, la ville, sa ville » menant en 1996 au projet " un projet culturel pour un projet urbain " (SYSTEME FRICHE THEATRE, 1996) réalisé avec l'aide d'un nouveau président emblématique: Jean Nouvel; (3) la capacité à se saisir d'opportunités de financement pour lancer des projets innovants (premier cybercafé de France en 1995, montage d'un projet de culture-multimédia (1996-98) financé par le ministère de la culture, création, en 1996, du premier Groupement d'Employeurs pour l'Insertion et la Qualification); (4) enfin un positionnement marqué sur les différents réseaux médiatiques grâce à l'appui sur les propres réseaux locaux, nationaux et internationaux dont les membres d'SFT et leur président font partie ainsi qu'une forte couverture médiatique, à tous niveaux (voir par exemple ACHMY, 1993; BEDARIDA, 1996 ; BUOB, 1995 ; SAMSON, 1997). Ce positionnement est d'ailleurs renforcé par la participation de Fabrice Lextrait, en tant que chargé de mission auprès du Secrétaire d'Etat au Patrimoine et à la décentralisation culturelle, Michel Duffour, à l'identification des nouveaux territoires de l'art via la publication du rapport "Friches, laboratoires, fabriques, squats, projets pluridisciplinaires » et l'organisation d'un colloque à la Friche en 2002. Ces stratégies sont similaires au Flon quoique plus locales. Dès 1992-1993, le réinvestissement du Flon est largement couvert par la presse lausannoise et vaudoise (voir par exemple BUSSY, 1993 ; BROU, 1992 ; JAUNIN, 1992 ; PECLET, 1994). Les locataires du Flon saisissent cette réceptivité favorable de la population et des médias pour accentuer leur emprise sur le site et tenter de pérenniser leur assise sur cette plate-forme soumise aux fluctuations des aspirations planificatrices de la Ville et du propriétaire. Ils usent en cela de divers outils. Ils se rassemblent en association, "l'Associaflon " qui fusionnera quelques années plus tard avec l'A.I.V.F, I'association historique des locataires du quartier, et créent un journal de quartier (la Gazette du Flon). Cette cohésion et ce dynamisme interne, renforcés par le laisser-faire du propriétaire et la non-intervention de la Ville, qui concentre toute son attention sur la réalisation d'un nouveau plan d'affectation, est favorable à l'émergence de ce "village » dans la ville, le "Flon-Flon ». En l'occurrence, les manifestations qui s'y déroulent, certaines plus privées comme des barbecues, d'autres plus ouvertes sur le monde extérieur (projection de films sur un des hangars visible sur la photo 2, organisation de fêtes) renforcent l'image alternative du site. Par ailleurs, comme à Marseille, les locataires parviennent à se mobiliser et à se faire entendre afin de s'opposer au projet Pont Ville, proposé et formalisé par la Ville suite à un concours lancé en 1987 et rejeté par refus d'entrée en matière ${ }^{\mathrm{vi}}$ en 1994 . Les locataires du Flon rejoignent à ce moment une autre association I'A.P.A.H.Fvii qui se mobilise contre le projet et propose d'ailleurs un contre- 
projet : "Flon Ville ou comment bâtir sans trop détruire " (A.P.A.H.F, 1993). L'association avec l'aide des locataires du Flon est prête à se mobiliser pour réclamer un référendum populaire. Pour preuve, à son opposition émise lors de l'enquête publique, elle joint une pétition de 2311 signatures (VILLE DE LAUSANNE, 1993). Cette position en faveur de la conservation de la structure et de l'âme du Flon rejoint celle des locataires du Flon qui tout en craignant pour le devenir du Flon-Flon souhaitent par la même préserver leur activité économique. Se greffent à eux des opposants politiques, une partie de la population locale et par extension le propriétaire qui ne trouve pas son compte financier dans le projet proposé par la Ville.

Or, la capacité des acteurs transitoires à bâtir leur stratégie de développement et leur manière de pérenniser leurs activités reposent sur la nature même de ces acteurs transitoires (leurs caractéristiques individuelles et collectives ainsi que celles de leurs activités). C'est d'ailleurs, en ce sens, qu'ils se distinguent de tous les autres acteurs temporaires (squatteurs par exemple) et qu'ils constituent le levier du changement d'image du site. Les nouveaux locataires du Flon par exemple ne sont pour autant pas novices dans leurs activités respectives. Certains d'entre eux bénéficient d'une véritable assise et visibilité dans leur domaine professionnel. Leur implantation au Flon constitue un véritable atout en vue de la consolidation et de la diffusion du "Flon-Flon ». Ce constat est particulièrement valable dans le domaine de l'art contemporain, avec les galeries Alice Pauli et Patrick Roy, connues internationalement, dans celui des activités de la nuit, avec le Moulin à Dances (MAD), qui fait partie des boîtes de nuit européennes accueillant les disc jockeys connus internationalement, ou encore dans le secteur vestimentaire branché, avec la boutique de vêtement Maniak de Fifi et Babette. L'image renvoyée par ces activités mais aussi par le public cible de ces enseignes, $c^{\prime}$ est-à-dire des jeunes branchés pour le MAD et la boutique Maniak, ou, pour les galeries, des collectionneurs, pratiquant ces lieux urbains à part comme à Londres ou à New York, renforce le caractère alternatif du site. Le MAD dès la fin des années 1980, occupe une place importante en Suisse, que ce soit en matière d'actions préventives contre le HIV ou d'accompagnement des revendications homosexuelles. Le magasin Maniak, quant à lui, dont la marque du même nom a été créée en 1983, cible une clientèle jeune, alternative, et aisée ; il bénéficie d'une véritable renommée en Suisse dans le milieu de la mode vestimentaire alternative et branchée. 


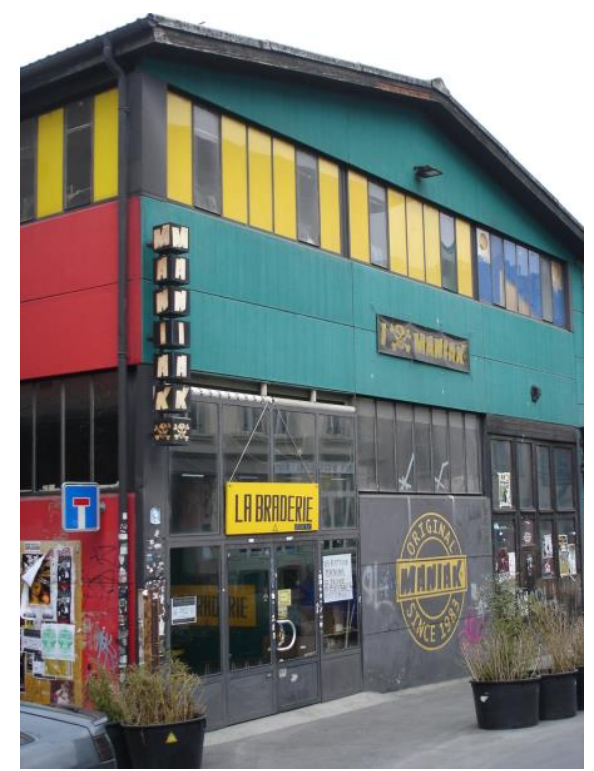

Photo 5 : L'entrepôt dans lequel s'installe le magasin Maniak (L. Andres, 2007)

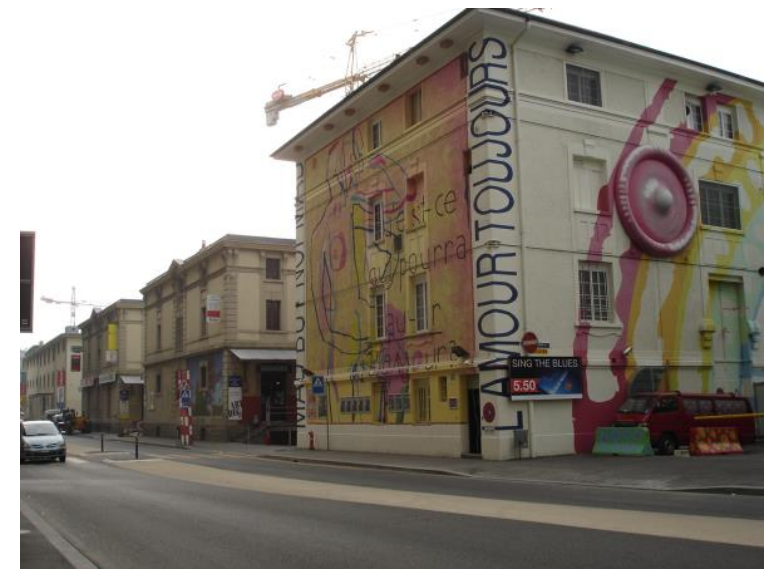

Photo 6 : Le MAD (L. Andres, 2007)

Les membres d'SFT sont également de véritables professionnels culturels. Philippe Foulquié, marseillais d'origine, déménage à Paris où il est successivement responsable puis directeur de centres culturels à Argenteuil et administrateur de compagnies indépendantes, comme le Théâtre de l'Unité. II devient dans les années 1980 le représentant du centre national du théâtre de marionnettes et travaille ensuite pour la DRAC à Cannes avant de créer, à la demande du ministère de la culture, le théâtre de marionnettes « Massalia » en 1987, à destination du jeune public. II revient ainsi à Marseille et est contacté par Christian Poitevin quelques années après. Tout juste diplômé d'un DESS de gestion d'entreprises culturelles et de développement local, Fabrice Lextrait fréquente le milieu culturel et associatif marseillais (par exemple Radio Grenouille). Il devient le bras droit de Philippe Foulquié et la cheville ouvrière du projet d'utilisation nomade des friches, proposé par Christian Poitevin. Administrateur de la Friche (jusqu'en septembre 2000), il devient chargé de mission pour 
Michel Duffour avant de rejoindre la direction d'Architectures Jean Nouvel en 2002. Outre ces deux piliers, SFT s'entoure également de personnes clés, Jean Nouvel puis Robert Guédiguian (comme présidents successifs), Ferdinand Richard et son association AMI (comme membre active de SFT dès l'origine), Armand Gatti ou IAM pour ce qui est des groupes d'artistes présents à la Friche. Tous ces professionnels œuvrent et formalisent la reconnaissance et la renommée du site. Ainsi, la place de ces acteurs, transitoires mais in fine pérennes, non décisionnaires mais partie prenante de la mutation, n'est pas anodine et due au hasard. Elle repose clairement sur plusieurs facteurs : (1) la solidité de ce système d'acteurs en d'autres termes leur structuration interne, (2) leur crédibilité (c'est-à-dire leur bagage professionnel conférant une reconnaissance sociale dans et à l'extérieur de leur milieu d'appartenance), (3) leur visibilité tant médiatique qu'au sein de réseaux territoriaux développés à différentes échelles, (4) leur intelligence sociale, soit leur capacité de négociation, d'entente ou de collaboration avec des personnages clés, ....) et, enfin, (5) leur force de proposition et de contre-proposition (ANDRES, 2008).

\section{3- L'illusion de la fossilisation de la friche et la nécessité d'une normalisation plus ou moins consensuelle : quelle leçons dégager?}

Comme évoqué précédemment, la perpétuation du contenu des activités (et du sens du lieu) durant le temps de veille est liée aux stratégies et aux projets développés par les acteurs transitoires qui, peu à peu, ne sont plus de simples acteurs transitoires et temporaires mais de véritables acteurs, porteurs d'intérêts (stakeholders à l'anglo-saxonne). Deux cas de figure peuvent être identifiés: (1) soit les acteurs transitoires acceptent une mutation qui semble tenir compte du temps de veille mais, pour certains d'entre eux, acceptent avec difficulté les évolutions dont le site fait l'objet (le Flon par exemple) ; (2) soit, malgré plusieurs tentatives menées en vue d'influencer la mutation globale du lieu (et pas uniquement du bâtiment ou du site dans lequel ils sont installés), les acteurs transitoires se ressoudent à se focaliser uniquement sur le développement et la mutation de l'ancienne friche (Friche de la Belle de Mai en l'occurrence). A Lausanne, la mutation du Flon et la votation du PPA passent par un encadrement des locataires du Flon qui constituent pour le Lo et la Ville une coalition d'opposants détenant, dans le contexte de démocratie participative helvétique, le pouvoir d'opposition vis-à-vis du projet et de mobilisation éventuelle de la population locale et des médias. Le propriétaire qui est véritable en charge, avec la ville, de la négociation du contenu du PPA de la Flon Vision met alors en place une stratégie poussée de dialogue et de communication qui prend appui sur différents supports et outils tels que des réunions avec les acteurs, des groupes de travail ou des plaquettes de 
communication (GROUPE LO, 1998) et des communiqués à la presse. Cette stratégie vise deux publics cibles : d'un côté, les locataires du Flon, via les groupes de travail, les réunions et, de l'autre, la population lausannoise et, par extension, la Ville et les acteurs privés (c'està-dire de nouveaux commerçants ou de nouvelles enseignes). Comme le résume le chargé de communication du groupe Lo de l'époqueviii, l'objectif affiché est clair : " trouver l'adhésion, la compréhension, des gens du Flon " et donc obtenir "les sympathies qu'ont ces gens, parmi la population de Lausanne, indispensables pour, d'une part, avoir suffisamment d'influence sur les autorités, et, d'autre part, convaincre, plus tard, les citadins de descendre au Flon ». La multiplication des outils de communication va bien au delà de la simple diffusion des informations. La mise en place de ces outils repose sur la stratégie du propriétaire : encadrer, maîtriser les revendications des locataires du Flon et s'attirer l'attention et l'intérêt des médias. Cette stratégie de communication relève d'une approche managériale poussée puisque la réalisation des groupes de travail, même s'ils ne fonctionnent pas très bien, permet d'anticiper et de tenter de réguler, à l'amiable, les facteurs d'opposition. C'est aussi un outil pour déstructurer les éventuelles coalitions d'opposants et mettre à l'écart les individus les plus critiques. Cette démarche de communication aboutit (puisque le PPA est voté) et se poursuit dans une stratégie de marketing urbain du Flon divisé en trois quartiers : "Saint Germain au Flon », "Flon-Flon », "Flon Business ». Chaque quartier est destiné à accueillir certaines fonctions et publicscible. D'où aujourd'hui un quartier abritant des restaurants et magasins branchés, des activités de loisirs (cinéma, boîtes de nuit ...) auxquelles s'ajoutent aussi les une grande partie des services municipaux (Flon-Ville) ainsi que quelques lofts. L'ensemble conduit à une gentrification économique du Flon qui tout en étant clairement orienté dans une démarche d'urban design et de différenciation architecturale (visible sur les photos 7 et 8 ) perd progressivement son côté alternatif caractéristique du Flon-Flon.

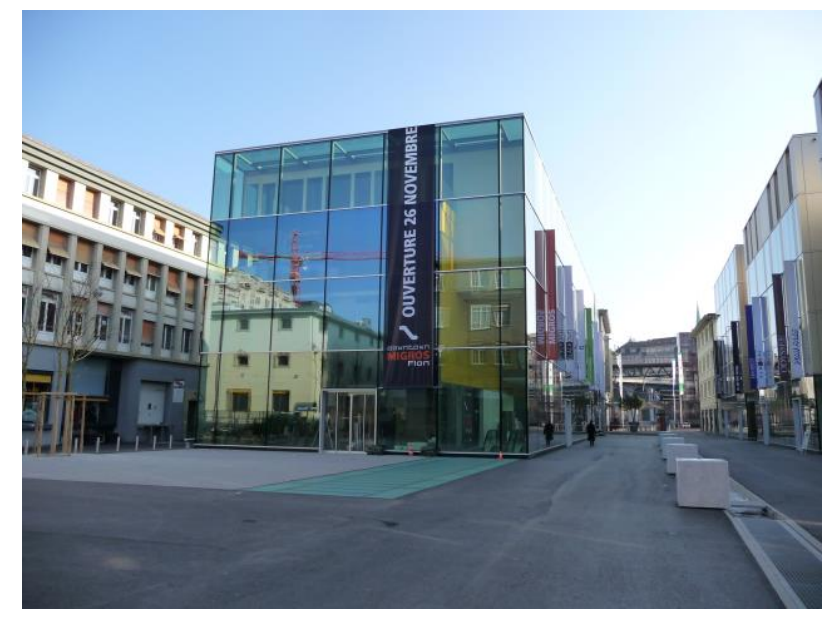

Photo 7 : Le Flon et son tissu urbain hétérogène (L.Andres, 2009) 


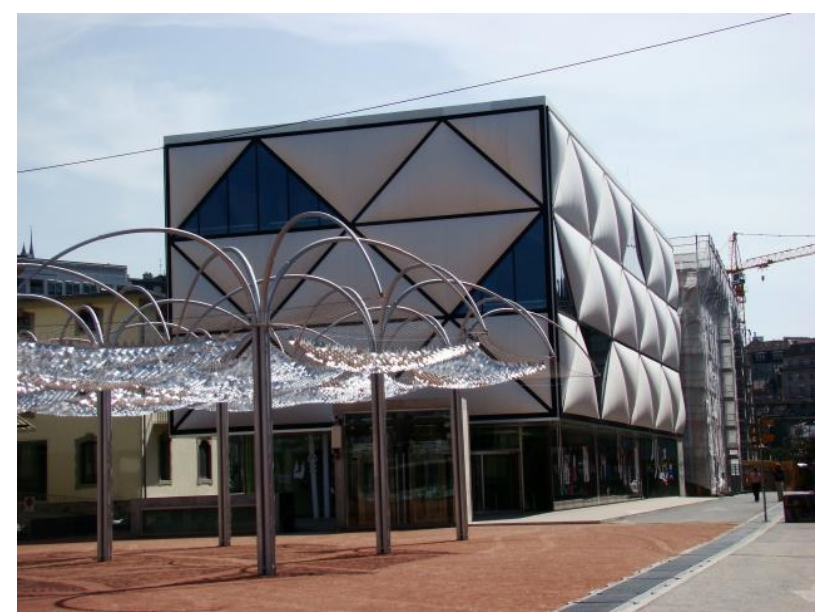

Photo 8 : Le bâtiment de la Miroiterie (L. Andres, 2008)

A la Belle de Mai au contraire, la pérennisation de la Friche passe par l'intervention et l'implantation des équipements publics sur les îlots 1 et2. Pour autant malgré les tentatives d'SFT aucune stratégie globale n'est mise en œuvre sur l'ensemble des trois îlots. La mutation de l'îlot 3 (la Friche) quant à elle traîne. Son achat par la Ville, à la Seita, en 1998 aboutit à l'adoption d'un schéma directeur en deux phases (2002 et 2005), "l'Air de ne pas y toucher " (TAUP, BOUCHAIN, SYSTEME FRICHE THEATRE, 2002 ; 2005) qui conduit véritablement aux premiers travaux de réhabilitation et de démolition-reconstruction. Par ailleurs, le statut juridique et administratif du lieu change et, ce, de manière innovante. La Friche est désormais régie par une $\mathrm{SCIC}$, la première $\mathrm{SCIC}$ culturelle en France, à laquelle la Ville a mis à disposition l'îlot 3 via un bail emphytéotique de 40 ans. La SCIC permet à la Friche d'être complètement autonome quant à la gestion et à l'évolution du site. Bien sûr, cette autonomie n'est pas sans risque mais elle peut permettre à la Friche de devenir une véritable entreprise culturelle. Elle constitue aussi une réponse financière pour la municipalité puisqu'elle vise à alléger sa participation économique et à promouvoir une meilleure implication des autres collectivités territoriales. Cette évolution positionne d'autant plus les enjeux financiers et de management stratégique de l'espace au cœur de l'action de SFT, ce qui ne va pas sans déplaire à certains locataires du lieu, loin d'adhérer à ces principes de fonctionnement. Ce changement de statut est pour autant garant de la perpétuation du projet et constitue un des points forts de la trajectoire de mutation réussie, et exceptionnelle, du lieu. 


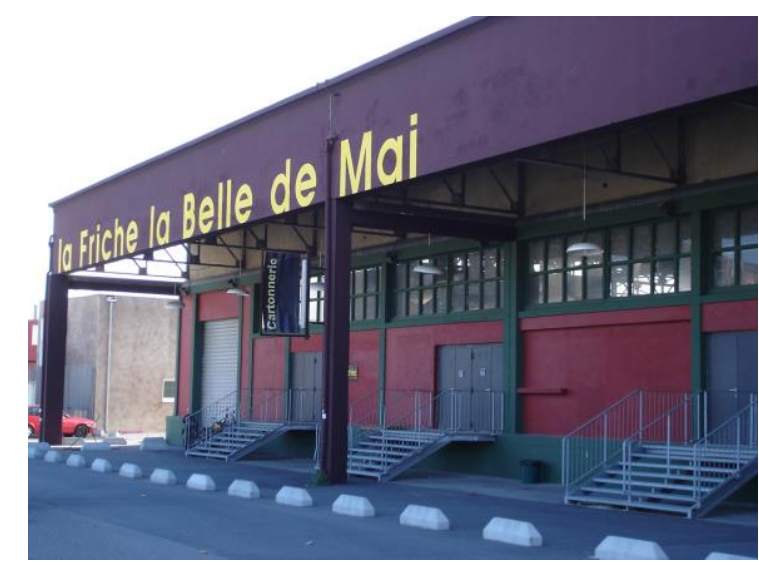

Photo 9 L'ancienne cartonnerie réhabilitée (L. Andres, 2008)

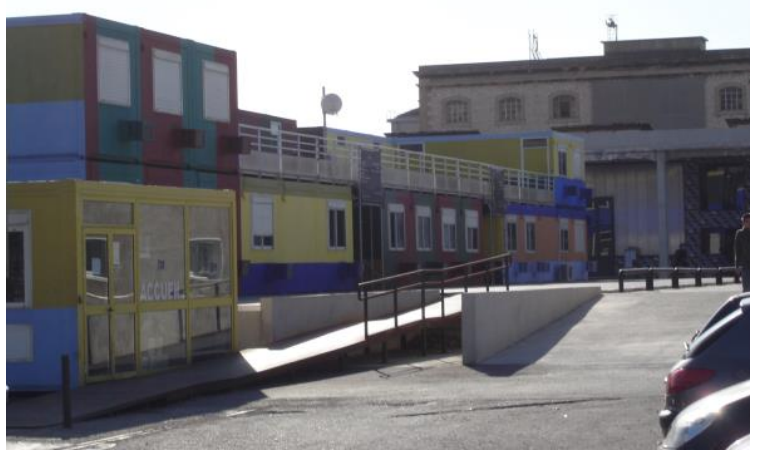

Photo 10 Nouveaux bureaux d'SFT et restaurant au fond (L. Andres, 2008)

Ainsi il est certain que c'est tout au long de ce cheminement menant à la réinsertion des espaces en friche dans de véritable documents de planification que se mettent en place les différentes stratégies des acteurs décisionnaires et-ou propriétaires passant par des outils de communication diversifiés et un encadrement plus ou moins autoritaire des coalitions d'opposants. Cela conduit donc à une continuité ou non entre le temps de veille et celui de la conception, propice au "croire " et au "faire croire ", et à celui de l'action, particulièrement vif au Flon. Une hiérarchie décisionnelle et foncière se créé alors entre les différents acteurs; celle-ci relève d'un processus d'acquisition d'une puissance d'action à la fois discursive (se faire entendre), matérielle (occuper l'espace), communicationnelle (être visible) et financière (avoir les moyens économiques). Clairement, même s'il n'y a pas de domination de principe d'un acteur sur les autres, plus la friche avance dans sa mutation, plus un groupe restreint d'acteurs acquiert un rôle durable sur la construction du processus de mutation. Dans le cas du Flon, cela conduit à des formes de gouvernance encadrée, voire contrôlée et planifiée. A la Belle de Mai, cela réside dans l'émergence d'un binôme d'acteurs, peu ouverts à la discussion, qui aboutit à la création de trois îlots déconnectés les uns des autres (hormis leur couleur culturelle), tournant le dos au quartier jointif de la Belle de Mai et allant à l'encontre d'un véritable projet urbain. Des lors il convient de dresser 
finalement un regard critique quant à l'impact de ces deux projets et de se poser la question suivante : le fondement de la perpétuation - plus ou moins artificielle - du contenu et des acteurs du temps de veille n'est-il pas allé a contrario de certaines dynamiques de revalorisation et $n$ 'a-t-il pas eu des effets pervers?

II est clair que ces processus de régénération, largement centrés sur les activités culturelles, artistiques et de loisir sont au cœur des stratégies de régénération car elles ont prouvé leur impact en tant que secteur économique, en tant que véritables "business » pour les villes (ZUKIN, 1995; HARVEY, 1989) et comme atout majeur pour la renaissance urbaine (EVANS, 2001; HALL, 2000 ; VERWIJNEN, LEHTOVUORI, 1999). C'est le cas à Lausanne et à Marseille même s'il convient de modérer les impacts positifs de ces expériences. Le développement et la promotion des industries créatives (à la Belle de Mai au travers du pôle multimédias sur l'îlot 2 et pour partie dans l'îlot3) et de la classe créative, décrite à maintes reprises par R. FLORIDA (2002), population cible du Flon-Flon et le nouveau Flon, sont partie prenante de la mutation de ces deux friches. Or, il a été souligné que Marseille a pris le contrepied des stratégies de régénération culturelles menées dans d'autres villes européennes à partir du moment où la municipalité a misé sur des expériences culturelles alternatives (nomadisme et politiques culturelles de C. Poitevin dans un premier temps) avant de se lancer dans la réalisation de vastes équipements vitrines (à l'image du Guggenheim et au travers du projet actuel du MUCEM en attente de construction au sein d'Euroméditerranée) pour contrecarrer les impacts de son déclin économique. A Lausanne au contraire c'est l'apport de solutions alternatives et non de projets grandiloquents et relativement traditionnels (Projet Pont Ville) qui a été au cœur du renouvellement du lieu. Or ces deux cas de figures contrecarrent pour partie les phénomènes similaires observables dans d'autres villes caractérisées par des quartiers culturels (par exemple Dublin's Temple Bar, Manchester's Northern Quarter, Bristol's harborside area, Sheffield's Creative Industries Quarter, the Quayside development in Newcastle) et ce pour la bonne raison que dans un cas comme dans l'autre, les activités développées durant le temps de veille et au-delà n'ont pas donné naissance à un véritable quartier culturel. De fait, les impacts à l'échelle du quartier ou vis-àvis des quartiers environnants sont plus que mitigés. Le quartier du Flon mute et se gentrifie économiquement au détriment de certains quartiers et commerces environnants et au plus grand dam des acteurs du Flon-Flon. Le quartier de la Belle de Mai quant à lui n'a que peu bénéficié du développement des trois îlots (BERTONCELLO, 2006 ; ANDRES, 2008); un milieu artistique composé de bars, de boîtes de nuit, typiques de quartiers culturels, ne s'est pas formé et greffé aux équipements culturels préexistants et déconnectés pour partie du réseau Friche (Théâtres Gyptis, Toursky et Bancs Publics). Seuls des petits commerces de proximité se sont installés ponctuellement. Le quartier de la Belle de Mai n'est ainsi pas 
marqué par l'arrivée d'une classe créative tirant profit des bâtis industriels disponibles pourtant propices dans certains cas à la production et à la recherche de matériaux (DRAKE, 2003); en aucun cas un milieu d'artistes ne s'est réellement créé et mis en réseau à la Belle de Mai comme observé dans certains quartiers culturels (MONTGOMERY, 1995; CREWE, BEAVERSTOCK, 1998). Ceci peut s'expliquer du fait que les locataires de la Friche n'ont pas forcément cherché à s'installer à proximité d'autant que le stock de friches était important dans toute la ville (et pas uniquement dans le quartier de la Belle de Mai); par ailleurs, le clivage tant fonctionnel que physique des trois pôles avec les espaces environnants a conforté la scission entre le quartier et l'ancienne manufacture. Ce clivage est d'ailleurs aussi institutionnel et au delà caractéristique du territoire marseillais. Non seulement le quartier et le pôle culturel de la Belle de Mai ne sont pas partie des même périmètres d'action (GPV, depuis 1999, et Euroméditerranée) mais, en plus, le maire du $3^{\text {ème }}$ arrondissement, dont fait partie le quartier, est membre de l'opposition et proche du président du Conseil Général (opposant politique au maire de Marseille). Enfin rien n'a été fait (à l'exception de quelques expériences ponctuelles menées par SFT ou les archives municipales) pour faire participer la population locale et ainsi tenter de tirer profit de la diversité, de l'histoire et des atouts du quartier (BOOTH, BOYLE, 1993; BIANCHINI, 1993 ; MILES, 2005 ; BROWN et al, 2000; BAILEY et al 2004) grâce à divers outils et processus participatifs. Typiquement, aucun organe de participation n'a accompagné la mutation des trois îlots. Au Flon, quartier à part entière, une fois le PPA voté, le Lo a été libre de valoriser et de tirer profit de son site et seuls désormais, ponctuellement, et de manière très légiférée, les locataires peuvent se faire entendre. D'où l'impression des anciens locataires de s'être fait avoir et de perdre tout pouvoir sur la mutation du site. "A l'époque, je me sentais bien sur $50000 m^{2}$, j'avais du plaisir à me promener, à saluer tout le monde et puis mon biotope s'est restreint au fur et à mesure. Ça fait 16 ans que je suis là. Avant j'avais une petite mer, après, j'ai eu un lac, ensuite une mare, et maintenant j'ai la rue là devant où je me sens à l'aise, encore pour quelques mois, car après quand il y aura les 3 bâtiments là devant, ce ne sera plus la même chose. Mais bon, tant que j'ai assez d'eau pour respirer je reste par là et puis après on ira ailleurs "ix.

Pour autant, bien que la mutation du Flon puisse laisser à désirer quant à sa pratique diurne et à la ségrégation socio-économique qu'elle appelle aujourd'hui, le quartier a néanmoins désormais retrouvé toute sa place dans la ville. Ceci découle pour partie du fait que le temps de veille a été considéré comme une composante majeure de la négociation et du socle du contenu du PPA mais, en aucun cas, comme critère déterminant et pérenne pour valoriser à moyen et long terme la plateforme. A la Belle de Mai, certes la trajectoire d'emprise d'SFT est exceptionnelle et traduit une capacité atypique de prise de possession de l'espace et de 
perpétuation d'une dynamique innovante de production d'une culture économie alternative, pour autant, ceci ne contribue en aucun cas à la réalisation d'un véritable projet urbain cohérent à l'échelle du quartier qui se limite à une juxtaposition d'îlots appelant nombre d'actions urbanistiques a-posteriori extrêmement problématiques (question de l'articulation morphologique entre l'ancienne usine et le quartier, gestion de l'accessibilité et des flux de circulation, etc.)

\section{Conclusion}

Ainsi donc, porter attention au temps de veille des friches permet de souligner la richesse des activités qui peuvent s'y développer et qui permettent ainsi d'amorcer la mutation de ces espaces leur conférant un nouvel usage et surtout une nouvelle image. De fait, au Flon comme à la Belle de Mai, le temps de veille est fondamental dans l'évolution de cet espace et dans sa réinsertion formelle - au sein de documents de planification- et grâce à l'action d'acteurs transitoires dont le rôle et le statut évoluent progressivement. Néanmoins, ce qui différencie la friche en veille d'autres espaces délaissés réside dans les stratégies et les projets développés par les mêmes acteurs transitoires afin de pérenniser leur présence et leurs activités (et par delà leurs projets). En d'autres termes un ensemble de facteurs, de pré-requis et de conditions préalables à la mutation ont pu être identifiés afin que le temps de veille se révèle être un véritable catalyseur de renouvellement. Néanmoins, ceci ne signifie pas pour autant une mutation sans heurts et satisfaisante tant pour les acteurs transitoires qu'en terme de cohérence urbanistique et sociale à une échelle (micro) locale. Cela n'enlève cependant pas l'attrait de ces lieux à l'échelle du territoire métropolitain et au delà, en tant par exemple que pôle ressource lors de la candidature de la Marseille au label capitale européenne de la culture (pour la Friche) ou que lieu d'attraction majeur pour le Flon. Diverses leçons peuvent finalement être dégagées : (1) l'importance de la capacité des acteurs extérieurs à procéder à une mise en lien des échelles territoriales et des acteurs en présence; (2) la nécessité et la prise de conscience de concilier les enjeux économiques et urbanistiques tout au long de la trajectoire de mutation; (3) l'adoption de formes de management à court et à long termes de projets non seulement par les acteurs décisionnaires mais aussi par les acteurs transitoires; (4) la promotion de véritables démarches partenariales fondées sur des relations gagnant-gagnant ; (5) l'attention portée à la qualité des acteurs en présence et à leur projet ancré dans la réalité économique, sociale et urbaine. 


\section{Bibliographie}

- ACHMY H., (1993), Donner une place aux artistes, L'humanité, 22-07-1993.

- A.P.A.H.F., (1993), Flon Ville ou comment bâtir sans trop détruire, CEDIPS, Lausanne, $23 \mathrm{p}$.

- ANDRES, L., (2008), La ville mutable. Mutabilité et référentiels urbains : les cas de Bouchayer-Viallet, de la Belle de Mai et du Flon, Thèse de Doctorat d'urbanisme et d'aménagement (Dir. Martin. Vanier), UMR Pacte-Territoire. Université Pierre Mendes France, Grenoble, 498p.

- BAILEY C., MILES S. et STARK, P., (2004), Culture-led urban regeneration and the revitalisation of identities in Newcastle, Gateshead and the north east of England, International Journal of Cultural Policy, vol.10, $\mathrm{n}^{\circ} 1$, p. 47-65.

- BEDARIDA, C., (1996), Les ruines urbaines renaissent grâce à la création artistique, Le Monde, 16-10-1996.

- BERTONCELlO B., (2006), La Friche de la Belle de Mai à Marseille : une vitrine dans un quartier populaire, Patrimoine de l'industrie, $n^{\circ} 2$, p. 59-68.

- BIANCHINI F., (1993), Culture, conflict and cities: Issues and prospects for the 1990s in BIANCHINI F. et PARKINSON M. (Eds), Cultural Policy and Urban Regeneration: The West European Experience, Manchester University Press, Manchester, p. 199-213.

- BOOTH P., BOYLE R., (1993), See Glasgow, see culture in BIANCHINI F. et PARKINSON M. (Eds), Cultural Policy and Urban Regeneration: The West European Experience, Manchester University Press, Manchester, p. 21-47.

- BROU F.H., (1992), Les défis de l'art contemporain au Flon, Scènes magazine, n59, pp. 58-59.

- BROWN A., O'CONNOR J. et COHEN, S., (2000), Local music policies within a global music industry: cultural quarters in Manchester and Sheffield, Geoforum, n ${ }^{\circ} 31, p$. 437-451.

- $\quad B U O B$, J., (1995), Marseille ou l'ambition artistique retrouvée, Le Monde, 11.01.1995.

- BUSSY A., (1993), Le Flon, centre de vie hétéroclite : des commerçants pleins d'espoir in Economie lausannoise, $\mathrm{n}^{\circ} 3$, pp. 4-5.

- CREWE L., BEAVERSTOCK J., (1998), Fashioning the city: Cultures of consumption in contemporary urban spaces, Geoforum, vol. 29, n³, p. 287-308.

- DONZEL A., (1998), Marseille : l'expérience de la cité, Anthropos, Paris, 193p.

- DRAKE G., (2003), This place gives me space: Place and creativity in the creative industries, Geoforum, n³4, p. 511-524.

- DUBOIS J., OLIVE M., (2004), Euroméditerranée : négociations à tous les étages, Les Annales de la recherche urbaine, $\mathrm{n}^{\circ} 97, \mathrm{p} .103-111$.

- EVANS G., (2001), Cultural Planning: An Urban Renaissance?, Routledge, London, $352 p$.

- FLORIDA R., (2002), The rise of the creative class: and how it's tranforming work, leisure, community and everyday life, Basic Books, New York, 416p.

- GROTH J., CORIJN E., (2005), Reclaiming Urbanity: Indeterminate Spaces, Informal Actors and Urban Agenda Setting, Urban Studies, vol.42, n³, p. 503-526.

- GROUPE LO, (1998), L'avenir du Flon, sans éd., n.p.

- HALL P. G., (2000), Creative cities and economic development, Urban studies, vol.37, p. 639-649. 
- HARVEY D., (1989), The Condition of Postmodernity: An Enquiry into the Origins of Cultural Change, Blackwell, Oxford, 320p.

- HATZFELD H., HATZFELD M., RINGART N., (1998), Quand la marge est créatrice : les interstices urbains initiateurs d'emploi, L'aube, Gémenos, 154p.

- HAYDN F., TEMEL R., (2006), Temporary Urban Spaces: Concepts for the Use of the City Spaces, Birkhauser, Basel, 272p.

- JAUNIN F., (1992), Coup de jeune à Lausanne sur Flon, Voir, n91, p. 41-42.

- MILES M., (2005), Interruptions: Testing the rhetoric of culturally led urban development, Urban Studies, vol.42, n5/6, p. 889-911.

- MINISTERE DES AFFAIRES SOCIALES, \& MINISTERE DE L'INTERIEUR ET DE L'AMENAGEMENT DU TERRITOIRE, \& MINISTERE DE L'EQUIPEMENT, (1993), Mission interministérielle : Marseille projet Joliette-Saint Charles, Paris, 106p.

- MONTGOMERY J., (1995), The story of Temple Bar: Creating Dublin's cultural quarter, Planning, Practice and Research, vol. 10, n², p. 135-171.

- OSWALT P., ( 2005), Shrinking cities, Hatje Cantz, Ostfildern-Ruit, 735 p.

- OVERMEYER K. (Eds), (2007), Urban Pioneers: Temporary Use and Urban Development in Berlin, Jovis, Berlin, 192p.

- PECLET J.C., (1994), Lausanne, le Flon : une victoire à courte vue, L'hebdo, 15-09-94, p. 73

- ROULLEAU BERGER L., (1995). La ville en friches : précarités, socialisations et compétences, Futur antérieur, 29/3.

- ROULleaU Berger, L., (1996), Cultures de friches à Marseille, Les Annales de la recherche urbaine, vol.70, p.16-25.

- ROULLEAU BERGER L., (1999), Le travail en friche : les mondes de la petite production urbaine. L'Aube, La Tour d'Aigues, 245p.

- SAMSON, M., (1997), Du groupe IAM au cirque Plume, en passant par les bières irlandaises Marseille, Le Monde, 08/07/1997.

- SANMARCO P., MOREL B., (1988), Marseille : l'état du futur, Edisud, Aix en Provence, $208 p$.

- SYSTEME FRICHE THEATRE, (1996), Friche la Belle de Mai : un projet culturel pour un projet urbain, Marseille, 39p.

- TAUP, BOUCHAIN P., SYSTEME FRICHE THEATRE, (2002), L'air de ne pas y toucher: assistance à maîtrise d'ouvrage auprès de la Ville de Marseille, Marseille, 43p.

- TAUP, BOUCHAIN P., SYSTEME FRICHE THEATRE, (2005), L'air 2 de ne pas y toucher: modification architecturale et urbaine de l'îlot 3 de la friche de la Belle de Mai, Marseille, 63p.

- URBAN UNLIMITED, (2004), The shadow city, Rotterdam, 66p.

- VERWIJNEN J., LEHTOVUORI P., (1999), Creative cities: cultural industries, urban development and the information society, UIAH publications, Helsinki, 272p.

- VILLE DE LAUSANNE, DIRECTION DES TRAVAUX, (1993), Plan partiel d'affectation au lieudit "Gare du Flon"in VILLE DE LAUSANNE, Bulletin du conseil communal : Débats au conseil communal relatif au projet ponts villes, séance du 6 sept 1994, Lausanne, p. 223-354.

- ZUKIN S., (1995), The Cultures of Cities, Blackwell, Oxford, 336p. 


\footnotetext{
i Société Coopérative d'Intérêt Collectif

ii Entretien Lauren Andres, Mars 2006

iii Entretien Lauren Andres, septembre 2005

iv Centre Interrégional de Conservation et de Restauration du Patrimoine

${ }^{\vee}$ Entretien Lauren Andres, Mars 2006

vi En Suisse, tout préavis est soumis à une première phase de discussion et de vote permettant de statuer sur le fait que le conseil communal accepte, ou non, de prendre en considération le préavis qui lui est soumis. Si l'entrée en matière est admise, la discussion sur le fond du préavis peut avoir lieu. Si elle est refusée, le préavis n'est pas discuté.

vii L'Association Pour un Aménagement Harmonieux de la vallée du Flon qui s'était déjà mobilisée lors du premier projet et avait contribué à son refus par référendum local

viii Entretien Lauren Andres

ix Entretien avec un des acteurs du Flon-Flon, Lauren Andres, septembre 2005
} 